\title{
Neurological morbidity and mortality associated with the endovascular treatment of cerebral arteriovenous malformations before and during the Onyx era
}

\author{
R. Webster Crowley, MD, ${ }^{1}$ Andrew F. Ducruet, MD, ${ }^{1}$ M. Yashar S. Kalani, MD, PhD, ${ }^{1}$ \\ Louis J. Kim, MD, ${ }^{2}$ Felipe C. Albuquerque, MD, ${ }^{1}$ and Cameron G. McDougall, MD, FRCSC ${ }^{1}$ \\ 1Division of Neurological Surgery, Barrow Neurological Institute, St. Joseph's Hospital and Medical Center, Phoenix, Arizona; \\ and 2 Department of Neurological Surgery, University of Washington, Seattle, Washington
}

\begin{abstract}
OBJECT The widespread implementation of the embolic agent Onyx has changed the endovascular management of cerebral arteriovenous malformations (AVMs). Recent data suggest that outcomes following embolization and resection may have worsened in the Onyx era. It has been hypothesized that there may be increased complications with Onyx embolization and increased surgical aggressiveness in patients treated with Onyx. In this study the authors analyzed their institutional experience with the endovascular treatment of cerebral AVMs prior to and after the introduction of Onyx to determine factors associated with periprocedural neurological morbidity and mortality.
\end{abstract}

METHODS A retrospective review was performed of all patients with cerebral AVMs undergoing embolization at the Barrow Neurological Institute from 1995 to 2012.

RESULTS Endovascular treatment of 342 cerebral AVMs was performed over 446 treatment sessions (mean age 37.8 years, range 1-83 years). Clinical presentation included hemorrhage in $47.6 \%$, seizures in $21.9 \%$, headaches in $11.1 \%$, and no symptoms in $10 \%$ of cases. The endovascular pretreatment strategy was preoperative in $78.9 \%$, preradiosurgery in $9.1 \%$, palliative in $5.3 \%$, targeted in $4.4 \%$, and curative in $2.3 \%$. The median Spetzler-Martin grade was III. The mean number of arteries embolized was 3.5 (range $0-13$ arteries), and the mean number of treatment sessions was 1.3 (range 1-4 sessions). Onyx was used in 105 AVMs (30.7\%), and N-butyl cyanoacrylate (NBCA) without Onyx was used in 229 AVMs (67\%). AVMs treated with Onyx had a higher mean number of arterial pedicles embolized than did NBCA cases $(4.3 \pm 2.7$ vs $3.2 \pm 2.4$, respectively; $p<0.001)$ and a greater number of sessions $(1.5 \pm 0.7$ vs $1.2 \pm 0.5$, respectively; $p<0.05$ ). Unexpected immediate postprocedural permanent neurological deficits were present in $9.6 \%$ of AVMs, while transient deficits were present in $1.8 \%$. There was 1 death $(0.3 \%)$. Spetzler-Martin grade was not associated with differences in outcome, as permanent neurological deficits were observed in $12 \%, 9 \%, 13 \%, 11 \%$, and $13 \%$ of AVMs for Spetzler-Martin Grades I-V, respectively $(p=0.91)$. The use of Onyx compared with NBCA was not associated with differences in periprocedural morbidity $(p=0.23)$. This lack of a difference persisted even when controlling for number of arteries and sessions $(p=0.14)$. Sex was not associated with differences in outcome.

CONCLUSIONS Permanent and transient postprocedural neurological deficits were noted in $9.6 \%$ and $1.8 \%$ of all cases, respectively. AVM grade was not associated with endovascular outcome. Despite the greater number of sessions required and arteries embolized for Onyx cases, there was no statistically significant difference in the risk of neurological deficits following cerebral AVM embolization with Onyx and NBCA.

http://thejns.org/doi/abs/10.3171/2015.2.JNS131368

KEY WORDS arteriovenous malformation; endovascular; $N$-butyl cyanoacrylate; Onyx; vascular disorders

ABBREVIATIONS AVM = arteriovenous malformation; $\mathrm{mRS}=$ modified Rankin Scale; NBCA = N-butyl cyanoacrylate . ACCOMPANYING EDITORIAL See pp 1490-1491. DOI: 10.3171/2014.9.JNS141419.

SUBMITTED June 26, 2013. ACCEPTED February 16, 2015.

INCLUDE WHEN CITING Published online March 27, 2015; DOI: 10.3171/2015.2.JNS131368.

DISCLOSURE Dr. Kim is an owner of, and patent holder for, SPI Surgical, Inc; has served as a consultant to Aesculap and Microvention; and has received clinical or research support for the study described from Volcano Inc. Dr. McDougall has served as a consultant to Covidien (eV3) and Terumo (Microvention). 
$\mathrm{T}$ HE treatment of cerebral arteriovenous malformations (AVMs) has been substantially altered with the development and refinement of endovascular techniques. As these techniques have been incorporated into the treatment paradigm for many AVMs, so too have the risks and benefits associated with endovascular treatment been incorporated into the overall risk profile for the treatment of AVMs. This is particularly important when endovascular treatment is performed prior to resection, as the risk-to-benefit ratio for preoperative embolization may be quite different for high-grade Spetzler-Martin AVMs when compared with Grade I AVMs. This has led some to conclude that the benefits of endovascular treatment for low-grade Spetzler-Martin AVMs are not worth the additional risks for what are typically low-risk surgical lesions. ${ }^{1}$

In 2006, our institutional experience with the endovascular treatment of cerebral AVMs was published, with specific emphasis on the neurological morbidity and mortality associated with embolization. ${ }^{2}$ Since that series was published, Onyx (eV3), a new embolic agent designed for the embolization of AVMs, has been approved and widely implemented for the endovascular treatment of cerebral AVMs. The implementation of this agent has been almost universally recognized as a step forward in the treatment of these complex vascular lesions. There have, however, recently been suggestions that the use of Onyx for the embolization of AVMs does not improve overall outcomes, and its use may even be associated with worse overall outcomes when compared with $N$-butyl cyanoacrylate (NBCA; Trufill, Cordis Neurovascular). ${ }^{5,6}$ It has been hypothesized that this may be a result of increased complications with Onyx embolization, or increased surgical aggressiveness in patients treated with Onyx. In this study we analyzed our institutional experience with the endovascular treatment of cerebral AVMs both prior to and after the introduction of Onyx to determine factors associated with treatment-related neurological morbidity and mortality.

\section{Methods \\ Study Population}

We reviewed our prospectively maintained database for every cerebral AVM treated endovascularly at Barrow Neurological Institute from June 1995 to January 2012. This time period represents every AVM embolized at Barrow Neurological Institute by 2 endovascular neurosurgeons (C.G.M. and F.C.A.). Embolization materials included Onyx, NBCA, detachable coils, liquid coils, embospheres, and polyvinyl alcohol (Boston Scientific). Approximately $95 \%$ of cases were treated under general anesthesia, with the remaining $5 \%$ performed in awake patients. No treatments were performed without general anesthesia after September 2000.

\section{Data Analysis}

Variables analyzed included age, sex, Spetzler-Martin grade, number of embolized arteries, number of embolization sessions, use of Onyx, use of NBCA, and use of other embolysates. Outcome variables included unexpected periprocedural neurological deficits, permanent unex- pected periprocedural neurological deficits, silent periprocedural complications, deaths, and total periprocedural complications. Total complications consisted of the sum of unexpected periprocedural neurological deficits and silent periprocedural complications. Periprocedural death occurred in only 1 patient and was not considered as an outcome variable in the analyses for this study. SpetzlerMartin grade was determined both by angiographic and noninvasive imaging.

\section{Statistical Analysis}

Associations between ordinal and categorical variables were assessed by chi-square tests. Independent sample ttests were used to examine group differences for continuous variables. ANOVAs were used to examine mean differences in number of sessions and number of arteries embolized by Spetzler-Martin grade. Post hoc analyses were corrected for using the Bonferroni method. A generalized binary logistic outcome model was used to examine differences in outcome by NBCA versus Onyx with covariates of number of sessions and arteries. SPSS (version 20, IBM) was used for all analyses.

\section{Results}

A total of 342 cerebral AVMs were treated endovascularly in 327 patients over 446 treatment sessions. Two patients were treated for multiple AVMs at different times, and 5 patients were treated for the same AVM multiple times in distinct, remote treatment periods. All but 1 of the AVMs $(341 / 342,99.7 \%)$ embolized during the study period were performed by the senior authors (C.G.M. and F.C.A.). The patients were $55.3 \%$ female $(n=189)$ and $44.7 \%$ male $(n=153)$, with an average age of 37.8 years (range 1-83 years). There were 56 patients (16.4\%) under the age of 18 years. The mean number of arterial pedicles embolized was 3.5 (range $0-13$ pedicles), and the mean number of embolization sessions was 1.3 (range $1-4$ sessions). The vast majority of AVMs were embolized in 1 or 2 sessions, comprising $76 \%$ and $19 \%$ of AVMs, respectively. Five percent of the AVMs were treated in 3 sessions, and less than $1 \%$ were embolized in 4 sessions. The mean clinical follow-up was 18.9 months (range 0-80 months).

AVM presentation included hemorrhage in $47.6 \%(\mathrm{n}=$ $163)$, seizures in $21.9 \%(\mathrm{n}=75)$, headaches in $11.1 \%(\mathrm{n}=$ 38), focal deficit in $4.9 \%(\mathrm{n}=17)$, and other AVM-related presentations in $4.4 \%(n=15)$. Ten percent $(n=34)$ of patients had asymptomatic AVMs that were incidentally discovered. The Spetzler-Martin AVM grades for the series were: $5 \%$ Grade I $(n=17), 26.6 \%$ Grade II $(n=91), 40.6 \%$ Grade III ( $\mathrm{n}=139), 20.8 \%$ Grade IV $(\mathrm{n}=71)$, and $7 \%$ Grade V $(n=24)$. The endovascular pretreatment strategy as determined from the medical records was preoperative in $78.9 \%(n=270)$, preradiosurgery in $9.1 \%(n=31)$, palliative in $5.3 \%(\mathrm{n}=18)$, targeted in $4.4 \%(\mathrm{n}=15)$, and curative in $2.3 \%(n=8)$.

Unexpected postprocedural neurological morbidity or mortality occurred in $11.4 \%(n=39)$ of AVMs. This was permanent in $9.6 \%(n=33)$, and transient in $1.8 \%(n=6)$ of all AVMs. There was 1 periprocedural death $(0.3 \%)$. Procedural complications that were clinically silent occurred in $7.6 \%$ of patients $(n=26)$. 


\section{Age and Sex Differences}

The mean age of patients treated with Onyx was lower than those treated without Onyx (mean $34.6 \pm 17.5$ vs 39.3 \pm 17.6 years old, respectively; $p<0.05$ ). There were no other variables with statistically significant differences detected for age or sex, including Spetzler-Martin grade and all of the outcome variables.

\section{Number of Embolization Sessions and Arteries}

The average number of sessions per AVM was higher for patients who encountered any type of complication when compared with those who did not have complications. This finding remained true for periprocedural neurological morbidity and permanent periprocedural neurological morbidity, but was not present for silent complications. Mean AVM sessions and standard deviations by outcome are presented in Table 1. The mean number of arteries embolized did not vary based on the presence or absence of any of the outcome variables.

\section{Embolysate Used}

Onyx was used as an embolysate in 105 AVMs (30.7\%). NBCA was used without concomitant Onyx in 229 AVMs (67\%), and was used in conjunction with Onyx in 39 AVMs (11.4\%). There were 4 cases in which NBCA was used expeditiously to manage vessel perforation in an Onyx treatment session, but for which it was otherwise not used. Four patients underwent attempted embolization but no embolysate was injected, largely due to the inability to adequately catheterize arterial pedicles. When compared with AVMs treated with NBCA, those treated with Onyx had a higher mean number of arterial pedicles embolized (mean $4.3 \pm 2.7$ vs $3.2 \pm 2.4$ pedicles, respectively; $\mathrm{p}<$ $0.001)$. In addition, the mean number of treatment sessions was higher with Onyx cases (mean $1.5 \pm 0.7$ vs $1.2 \pm 0.5$ sessions, respectively; $\mathrm{p}<0.05$ ).

Unexpected periprocedural neurological morbidity was noted in $13.1 \%$ of NBCA cases and $8.6 \%$ of Onyx cases, but this difference was not statistically significant $(\mathrm{p}=$ 0.23). Similarly, we were unable to confirm an association between unexpected permanent morbidity that was present in $11 \%$ of the NBCA cases compared with $8 \%$ of the Onyx cases $(\mathrm{p}=0.40)$, silent complications present in $7 \%$ of NBCA cases and 9\% of Onyx cases ( $\mathrm{p}=0.65)$, or total complications present in $20 \%$ of NBCA cases versus $17 \%$ of Onyx cases $(\mathrm{p}=0.56)$.

To determine if the average number of embolization sessions or arteries acted as a confounding variable in the association between Onyx usage and our outcome vari-

TABLE 1. Number of sessions per AVM by complication

\begin{tabular}{lccc}
\hline \multicolumn{1}{c}{ Outcome } & Complication* & $\begin{array}{c}\text { Without } \\
\text { Complication* }\end{array}$ & $\begin{array}{c}p \\
\text { Value }\end{array}$ \\
\hline Periprocedural morbidity & $1.54 \pm 0.76$ & $1.27 \pm 0.65$ & 0.04 \\
\hline Permanent morbidity & $1.58 \pm 0.79$ & $1.28 \pm 0.56$ & 0.04 \\
\hline Silent complication & $1.46 \pm 0.76$ & $1.29 \pm 0.57$ & 0.27 \\
\hline Total complications & $1.57 \pm 0.75$ & $1.26 \pm 0.53$ & 0.01 \\
\hline
\end{tabular}

* Data given as mean \pm SD. ables, 4 binary logistic regression models were run with embolization sessions and arteries included as predictor variables. Onyx usage failed to significantly predict outcome for any of our 4 models: periprocedural morbidity ( $\mathrm{p}$ $=0.14$ ), permanent morbidity ( $p=0.23$ ), silent complications $(\mathrm{p}=0.68)$, and total complications $(\mathrm{p}=0.35)$.

Other embolic materials (such as detachable coils, liquid coils, polyvinyl alcohol, and embospheres) were used in 49 AVMs (14.3\%), 40 of which were used in conjunction with NBCA, 5 in conjunction with Onyx, and 4 as the sole treatment. There was no statistically significant difference in outcomes for AVMs that used these other embolic materials when compared with those that did not. Total complications were observed in $24.5 \%$ of cases with other embolysates versus $18.1 \%$ without additional materials $(p=0.29)$. Similarly, there were no statistically significant differences detected in the other outcome variables of unexpected periprocedural neurological deficits $(16.3 \%$ vs $10.6 \%, \mathrm{p}=0.24)$, permanent unexpected periprocedural neurological deficits $(12.2 \%$ vs $9.2 \%, \mathrm{p}=$ $0.51)$, and silent periprocedural complications ( $8.2 \%$ vs $7.5 \%, \mathrm{p}=0.87)$.

\section{Spetzler-Martin Grade}

Spetzler-Martin grade was not associated with differences in total periprocedural complications, unexpected periprocedural neurological deficits, permanent unexpected periprocedural neurological deficits, silent periprocedural complications, total complications, or death (Table 2). Permanent neurological deficits were observed in $12 \%$, $9 \%, 13 \%, 11 \%$, and $13 \%$ of AVMs for Spetzler-Martin Grades I-V, respectively $(\mathrm{p}=0.91)$.

A statistically significant difference emerged in the number of arterial pedicles embolized by Spetzler-Martin grade $(\mathrm{p}<0.001)$. Post hoc analyses revealed that SpetzlerMartin Grade V patients on average had a higher number of arterial pedicles embolized than did Grade I ( $\mathrm{p}=$ $0.006)$, Grade II ( $p<0.001)$, or Grade III patients ( $p=$ 0.006). Spetzler-Martin Grade IV patients had a higher number of arterial pedicles embolized than did Grade II patients $(\mathrm{p}=0.002)$.

The number of embolization sessions also varied significantly by Spetzler-Martin grade $(\mathrm{p}<0.001)$. Post hoc analyses demonstrated that Spetzler-Martin Grade IV and $\mathrm{V}$ patients, on average, had more sessions when compared with Grades I, II, and III ( $\mathrm{p}<0.001$ for all 6 comparisons), and Grade III patients had more sessions when compared with Grade II patients $(\mathrm{p}=0.017)$.

\section{Discussion}

In 2005, Onyx, an ethylene-vinyl alcohol copolymer, was approved for the endovascular treatment of AVMs in the US. Since that time, it has become widely accepted and implemented by neurointerventionalists involved in the treatment of cerebral AVMs.

In 2006, we reported the results of our institutional experience with the embolization of 153 AVMs from 1995 to 2004,1 year prior to the FDA approval of Onyx. ${ }^{2}$ The vast majority of these patients $(144 / 153,94.1 \%)$ were treated with NBCA alone or in combination with another em- 
TABLE 2. Association between outcome measures and Spetzler-Martin grade

\begin{tabular}{lcccccc}
\hline & \multicolumn{5}{c}{ Spetzler-Martin Grade (\%)* } & \\
\cline { 2 - 6 } \multicolumn{1}{c}{ Outcome Measure } & $\mathrm{I}$ & $\mathrm{II}$ & $\mathrm{III}$ & $\mathrm{IV}$ & $\mathrm{V}$ & $\mathrm{p}$ Value \\
\hline Periprocedural morbidity & $2(5.1)$ & $8(20.5)$ & $18(46.2)$ & $8(20.5)$ & $3(7.7)$ & 0.91 \\
\hline Permanent morbidity & $2(6.1)$ & $7(21.2)$ & $16(48.5)$ & $5(15.2)$ & $3(9.1)$ & 0.77 \\
\hline Silent complication & $1(3.8)$ & $7(26.9)$ & $10(38.5)$ & $5(19.2)$ & $3(11.5)$ & 0.92 \\
\hline Total complications & $3(4.6)$ & $15(23.1)$ & $28(43.1)$ & $13(20.0)$ & $6(9.2)$ & 0.90 \\
\hline Mean no. of AVM sessions \pm SD & $1.00 \pm 0.00$ & $1.04 \pm 0.21$ & $1.27 \pm 0.55$ & $1.58 \pm 0.65$ & $1.88 \pm 0.99$ & $<0.001$ \\
\hline $\begin{array}{l}\text { Mean no. of arterial pedicles per } \\
\text { AVM session } \pm \text { SD }\end{array}$ & $2.59 \pm 1.97$ & $2.82 \pm 1.85$ & $3.38 \pm 2.28$ & $4.28 \pm 2.92$ & $5.25 \pm 3.63$ & $<0.001$ \\
\hline
\end{tabular}

* Numbers in parentheses are the percentages of the total for each particular outcome measure that occurred by grade.

bolysate. The periprocedural morbidity and mortality was $11.8 \%$, with 1 death. Seventeen patients suffered unexpected neurological deficits after treatment, 5 of whom went on to attain complete or near-complete recovery. Only $2.7 \%$ of patients suffered death or severe disability (modified Rankin Scale [mRS] score $\geq 2$ ) as a result of embolization. The study found that embolization of 3 or more branches was associated with increased risk of complications. In addition, there was a trend toward increased complications with increasing Spetzler-Martin grade. These previously reported patients were included in the current study and constitute the majority of the pre-Onyx era patients.

A number of other institutional series have been published investigating the results of the endovascular treatment of AVMs, but these series have typically not incorporated cases treated before and after the implementation of Onyx, or have not had sufficient numbers of Onyx or NBCA to perform a comparison. In 2004, Taylor et al. reported their experience from 1992 through 2003, when they treated 201 patients over 339 sessions.9 The majority of the AVMs were embolized using polyvinyl alcohol particles, while only $13 \%$ included NBCA, and 5 procedures $(1.5 \%)$ included Onyx. Death or permanent neurological morbidity was observed in $11 \%$ of patients, and no predictive variables were identified. In 2009, Starke et al. published their institutional results with the endovascular treatment of AVMs using NBCA. ${ }^{8}$ Their series consisted of 202 patients who underwent embolization as a precursor to resection or radiosurgery. These authors observed new clinical deficits in $14 \%$ of patients and persistent deficits in $2.5 \%$ of patients. They found that predictors of new neurological deficit following embolization were complex AVM requiring more than 1 session, AVM diameter $<3$ $\mathrm{cm}$, AVM diameter $>6 \mathrm{~cm}$, deep venous drainage, and eloquent location. Lv et al. reported on 118 patients who received partial treatment for brain AVMs using both Onyx and NBCA. These authors investigated subsequent annual risk of hemorrhage and found no significant difference in those treated with NBCA or Onyx. ${ }^{4}$

Recently Saatci et al. published their large series of 350 AVMs treated endovascularly with Onyx. ${ }^{7}$ Due to the earlier availability of Onyx in Turkey, their series spanned from 1999 to 2008, and while every patient was treated with Onyx, an additional $12 \%$ were also treated with NBCA for those cases with high-flow fistulas, dissections, or perforations. These authors achieved angiographic cure in $51 \%$ of patients, with an additional $38 \%$ requiring posttreatment radiosurgery. AVM recanalization was observed in $1.1 \%$ of the AVMs believed to be initially cured. The rate of permanent morbidity or mortality was $8.5 \%$, with a total of 5 deaths.

The only direct comparison of Onyx and NBCA for the preoperative treatment of cerebral AVMs was published in 2010 by Loh and Duckwiler ${ }^{3}$ This was an industrysupported equivalence trial that was conducted from 2001 to 2003, with the express purpose of obtaining FDA approval. The study included 54 patients treated with Onyx and 63 treated with NBCA, and the authors concluded that Onyx and NBCA were equivalent with regards to AVM volume reduction $\geq 50 \%$, as well as serious adverse events, resection time, and intraoperative blood loss. While the small sample size did not allow for the detection of a statistically significant difference, the rate of serious adverse events for AVMs treated with Onyx was almost double that of NBCA cases (9.2\% vs $4.8 \%$, respectively). Conversely, rates of technical and procedural complications were slightly lower with Onyx compared with NBCA ( $40.7 \%$ vs $46 \%$, respectively), a difference that again did not reach statistical significance. It is noteworthy that these complication rates include the learning curve associated with the introduction of Onyx in the trial. Additionally, techniques and devices used with Onyx have evolved considerably since completion of the trial. Technical nuances that have been described include the creation of a proximal plug using the more viscous Onyx 34, followed by embolization through the same catheter with Onyx 18, and the use of dual catheter techniques. Advancements in devices have also been made, such as detachable-tipped catheters and balloon-tipped dual lumen catheters that will hopefully increase the efficacy and decrease the risks of embolization.

Recently, however, a single-institutional experience was presented in which the authors compared surgical results of AVMs treated with preoperative embolization prior to Onyx, and with preoperative embolization using Onyx..$^{5,6}$ Analyzing 538 cases over a 19 -year period, the authors concluded that resection of Spetzler-Martin Grade I and II AVMs carries a risk of new permanent neurological deficit with an mRS score greater than 2 in $0.3 \%$ of cases. Grade IV and V AVMs were more likely to be embolized with Onyx than in the period prior to Onyx ( $80 \%$ of cases vs $43 \%$ of cases, respectively; $p=0.006$ ), 
and the authors reported that Onyx cases were more likely to result in an mRS score greater than 2 (35\% vs $12.2 \%$, respectively; $\mathrm{p}=0.03),{ }^{5}$ although this was not found to be statistically significant in their subsequent publication (30\% vs $12 \%$, respectively; $\mathrm{p}=$ nonsignificant). ${ }^{6}$ The authors concluded that the introduction of Onyx has not improved outcomes, and may embolden the neurosurgeon by giving a false sense of protection from complications. No data were presented relating to endovascular complications.

It is important to appreciate that, regardless of the embolic agent employed, it is likely that complication rates will vary with treatment objectives, operator experience, and operator perception of risk-to-benefit ratio. For example, if the goal of embolization is to facilitate resection, then the techniques employed will be quite different than if the goal is cure by embolization alone.

The rapid evolution of endovascular techniques and devices presents a constant challenge to the practicing physician. Endovascular devices and techniques have seen substantial changes over the past 10 to 20 years. This becomes more apparent, and problematic, when assessing the outcomes of patients treated with endovascular methods. Certainly long-term follow-up is necessary to accurately determine the risks and benefits of a procedure. However, in the case of the endovascular treatment of AVMs, particularly in the presurgical setting in which a patient will often be undergoing surgery within a day or two of embolization, it may be difficult to ascertain which complications were a result of the embolization and which were caused by surgery. In other words, there may only be a short period to determine the complications following embolization, and if a postembolization neurological deficit does not resolve over time, it may be difficult to ascertain whether the deficit was made permanent by surgery, or if it would have been permanent from the embolization alone. In addition, it is extremely difficult to determine if a complication attributed to endovascular treatment would have occurred as a result of surgery, even in the absence of embolization. For instance, a visual field deficit detected following embolization of an occipital AVM may have also occurred with surgery alone. For the purposes of our study, these complications were attributed to endovascular treatment. Based on our evaluation, with respect to the rate of complications that result from the embolization of AVMs, the choice of NBCA versus Onyx does not appear to be important. This raises the question of what other factors are therefore important in making the choice between the embolic agents, and highlights the difficulties inherent in evaluating the effectiveness of embolization. If the goal of embolization is cure, this can be evaluated objectively with relative ease. If, however, as in our institution, the primary objective for most patients is the facilitation of resection, then the effectiveness is best judged by the surgeon resecting the malformation. It is recognized that this is a subjective evaluation, as objective end points such as resection time and blood loss have not been reliable at discriminating between embolic agents.

Additionally, given that effectiveness was not evaluated in this study, it is entirely plausible that the embolizations were more effective in one era of this study versus the other, but that this better effectiveness was accomplished without an increase in the complication rate. Ultimately, the evaluation of effectiveness relative to the complication rate is the important consideration. This evaluation is much more difficult, and in the case of preoperative embolization, the effectiveness can only be judged in conjunction with the entire treatment plan. Combination therapy with preoperative embolization followed by surgery (or radiosurgery) can be viewed as a dynamic exercise in "risk sharing" between the procedures and optimal partitioning of this treatment risk to maximize successful treatment, while minimizing the overall complication rates is best accomplished by an experienced cohesive team that is able to dispassionately examine the impact of their treatment strategy. At present, we believe that in general we are able to achieve a greater degree of embolization with Onyx without increasing morbidity. We strongly believe, however, that NBCA does have a role in these procedures, particularly with high-flow fistulas.

\section{Conclusions}

In this patient series, we observed permanent and transient postprocedural neurological deficits in $9.6 \%$ and $1.8 \%$, respectively, of all cases that underwent endovascular treatment of cerebral AVMs. Patients who experienced complications had a higher mean number of embolization sessions than those without complications. SpetzlerMartin AVM grade was not associated with endovascular outcome. Finally, there was no statistically significant difference between Onyx and NBCA in regard to any of the outcome variables.

\section{References}

1. Hamilton MG, Spetzler RF: The prospective application of a grading system for arteriovenous malformations. Neurosurgery 34:2-7, 1994

2. Kim LJ, Albuquerque FC, Spetzler RF, McDougall CG: Postembolization neurological deficits in cerebral arteriovenous malformations: stratification by arteriovenous malformation grade. Neurosurgery 59:53-59, 2006

3. Loh Y, Duckwiler GR: A prospective, multicenter, randomized trial of the Onyx liquid embolic system and N-butyl cyanoacrylate embolization of cerebral arteriovenous malformations. Clinical article. J Neurosurg 113:733-741, 2010

4. Lv X, Wu Z, Li Y, Yang X, Jiang C: Hemorrhage risk after partial endovascular NBCA and ONYX embolization for brain arteriovenous malformation. Neurol Res 34:552-556, 2012

5. Morgan MK: The failure of preoperative ethylene-vinyl alcohol copolymer embolization to improve outcomes in AVM management: case series, in The American Academy of Neurological Surgery $73^{\text {rd }}$ Annual Meeting, Scottsdale, Arizona, October 19-22, 2011. Park Ridge, IL: American Association of Neurological Surgeons, 2011

6. Morgan MK, Davidson AS, Koustais S, Simons M, Ritson EA: The failure of preoperative ethylene-vinyl alcohol copolymer embolization to improve outcomes in arteriovenous malformation management: case series. J Neurosurg 118:969-977, 2013

7. Saatci I, Geyik S, Yavuz K, Cekirge HS: Endovascular treatment of brain arteriovenous malformations with prolonged intranidal Onyx injection technique: long-term results in 350 
consecutive patients with completed endovascular treatment course. J Neurosurg 115:78-88, 2011

8. Starke RM, Komotar RJ, Otten ML, Hahn DK, Fischer LE, Hwang BY, et al: Adjuvant embolization with N-butyl cyanoacrylate in the treatment of cerebral arteriovenous malformations: outcomes, complications, and predictors of neurologic deficits. Stroke 40:2783-2790, 2009

9. Taylor CL, Dutton K, Rappard G, Pride GL, Replogle R, Purdy PD, et al: Complications of preoperative embolization of cerebral arteriovenous malformations. J Neurosurg 100:810-812, 2004

\section{Author Contributions}

Conception and design: McDougall, Crowley. Acquisition of data: Ducruet, Kalani, Kim. Analysis and interpretation of data: Ducruet, Kim. Critically revising the article: McDougall, Ducruet, Albuquerque. Reviewed submitted version of manuscript: McDougall, Crowley, Ducruet, Albuquerque. Statistical analysis: McDougall, Crowley. Study supervision: McDougall, Albuquerque.

\section{Supplemental Information}

Current Affiliation

Dr. Crowley: Division of Neurosurgery and Radiology, University of Virginia Medical Center, Charlottesville, VA.

\section{Correspondence}

Cameron G. McDougall, c/o Neuroscience Publications, Barrow Neurological Institute, 350 W. Thomas Rd., Phoenix, AZ 85013. email: neuropub@dignityhealth.org. 